\title{
COMPOUNDS EXHIBITING PROLONGED ANTIOESTROGENIC AND ANTIFERTILITY ACTIVITY IN MICE AND RATS
}

\author{
G. W. EMMENS \\ Department of Veterinary Physiology, University of Sydney, \\ Sydney 2006, Australia
}

(Received 4th September 1970, revised 15th December 1970)

Summary. Various triarylalkene derivatives which are weakly oestrogenic and not antioestrogenic at any dosage in conventional tests in mice and rats cause refractoriness to oestradiol for several weeks after injection in vaginal smear tests.

The same compounds, administered in a single injection of $1 \mathrm{mg}$ before mating, caused complete sterility for up to several weeks, although mating still occurred, sometimes at a slower rate than in controls. Daily injections of effective doses ( 5 to $50 \mu \mathrm{g}$ ) produced similar effects, but reduced mating frequencies more severely.

Oral administration was not followed by refractoriness to oestradiol. When a single dose of $1 \mathrm{mg}$ was fed however, mating was normal but fertility was reduced by up to $85 \%$ compared with controls. Daily oral dosing in the vehicle used (50\% aqueous propylene glycol) or even of the vehicle alone upset the animals and reduced both mating and fertility.

\section{INTRODUGTION}

Since Lerner, Holthaus \& Thompson (1958) described MER-25 (1-[ $p$-(2diethylaminoethoxy)phenyl]-2-( $p$-methoxyphenyl)-1-phenylethanol), various triarylethane and triarylethylene derivatives with basic ether groups have been found to possess both antioestrogenic and antifertility activity (see Emmens, 1970 , for a recent review). Antioestrogenic activity has usually been detected by administering an oestrogen and potential antagonist simultaneously in uterine weight or vaginal smear tests, and antifertility activity by administering the compounds before or shortly after coitus. For example, MRL-37 (1-[p(2-diethylaminoethoxy) phenyl]-2-(p-methoxyphenyl)-1-phenylethane) prevents conception in rodents whether given before, or on Days 1 to 3 or 4 to 6 , after coitus (Emmens, Humphrey, Martin \& Owen, 1967).

Harper \& Walpole (1966) showed that the cis- and trans- isomers of 1 [ $p$-(2dimethylaminoethoxy)phenyl]-1,2-diphenylbut-1-ene have different properties. The cis-isomer, ICI-47,699, is a potent oestrogen in rats and mice, without antioestrogenic activity at any dosage level, and appears to act as a typical oestrogen in causing failure of implantation when given after coitus. The 
trans-isomer, ICI-46,474, is an even more potent oestrogen in the mouse, still not antioestrogenic in that species, and highly potent as an antifertility agent, whereas, in the rat, it is a weak oestrogen, a potent antioestrogen, and also a highly potent antifertility agent after coitus.

These results were largely confirmed by Collins, Hobbs \& Emmens (1971) who examined a series of similar compounds, often characterized fully with respect to their stereoisomerism. In general, such compounds do not function as antioestrogens when injected into the mouse, but are weakly oestrogenic, often with a very low slope to the dose-response line, and are highly potent as antifertility agents. Exceptions, however, were found, showing weak subcutaneous antioestrogenicity accompanied in one case by no antifertility action and in the other by fairly potent antifertility activity after coitus. For the most part, these compounds showed no convincing correlation of either oestrogenic or antioestrogenic activity with antifertility properties, when their activities were measured as described above.

It was therefore with some surprise that it was observed that in vaginal smear tests, interference with responses was occurring in animals previously dosed with triarylethylene derivatives. Particularly after subcutaneous administration, the higher doses of some compounds caused refractoriness to oestradiol and other oestrogens, even to the oestrogenic effects of the compound originally administered. This paper gives preliminary results of an investigation into the phenomenon, and into antifertility effects which were found to accompany it.

\section{MATERIALS AND METHODS}

Vaginal smear tests were conducted in QS mice or albino rats as described by Emmens (1969), with two injections or feedings in oil or $50 \%$ aqueous propylene glycol respectively on the mornings of consecutive days. Smears were taken on the evening of the 3 rd day and morning of the 4th day, stained in $5 \%$ methylene blue and scored as positive or negative. The total percentage score for each group, usually of ten mice, is used in the Tables, each smear contributing one unit. When oestrogen and an experimental compound were administered simultaneously, each was given by separate injection, or one by injection and one orally.

Fertility tests were conducted by injecting or feeding a single dose of a compound to female mice 3 days before introducing males, with twenty mice per group. The mice were examined daily and segregated on the discovery of a vaginal plug. In other tests, daily injection or oral dosage was started 3 days before introducing the males and continued until mating occurred or for 10 days, whichever was the sooner.

The compounds used in these tests were all triarylalkene derivatives of the compounds shown in Table 1.

\section{RESULTS}

Vaginal smear tests

The first substance investigated in some detail was H-774 (cis, trans mixture, 
about $85 \%$ trans) and the corresponding isomers, $\mathrm{H}-1067$ (trans) and $\mathrm{H}-1071$ (cis). This experiment started with a vaginal smear test as described above, using the experimental compounds. At 2-week intervals thereafter, further vaginal smear tests were made on the same animals, using a total dose of $0.1 \mu \mathrm{g}$ of oestradiol-3-17 $\beta$ which, on its own, would give almost $100 \%$ of positive responses. The results are shown in Table 2. Responses to the experimental compounds are irregular (Collins et al., 1971), but a high proportion of positive smears indicates the weak and atypical oestrogenicity seen with these compounds in an Allen-Doisy test. Two weeks later, the response to oestradiol was abolished in mice which had received the higher doses of $\mathrm{H}-774$ and $\mathrm{H}-1067$ by injection $(P<0.01)$; the cis-compound, $\mathrm{H}-1071$ had a weaker effect. No significant reduction in responses to oestradiol occurred in mice receiving these

TABLE 1

THE COMPOUNDS INVESTIGATED

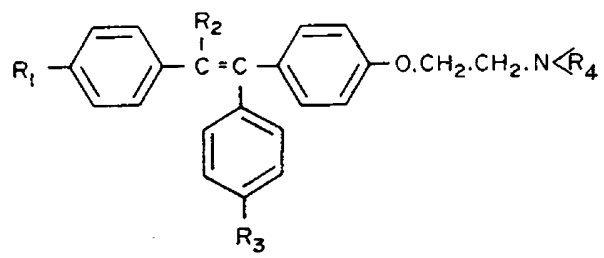

\begin{tabular}{|c|c|c|c|c|}
\hline Compound & $\mathrm{R}_{1}$ & $\mathbf{R}_{\mathbf{2}}$ & $\mathbf{R}_{3}$ & $\mathbf{R}_{\mathbf{4}}$ \\
\hline $\begin{array}{l}\text { IGI-46,474 } \\
\text { ICI-47,699 } \\
\text { ICI-45,960 } \\
\text { ICI-45,692 } \\
\text { H-774 } \\
\text { H-1067 } \\
\text { H-1071 } \\
\text { H-1075 } \\
\text { H-1076 }\end{array}$ & $\begin{array}{l}\mathrm{H} \\
\mathrm{H} \\
\mathrm{H} \\
\mathrm{H} \\
\mathrm{OCH}_{3} \\
\mathrm{OCH}_{3} \\
\mathrm{OCH}_{3} \\
\mathrm{OCH}_{3} \\
\mathrm{OCH}_{3}\end{array}$ & $\begin{array}{l}\mathrm{C}_{2} \mathrm{H}_{5} \\
\mathrm{C}_{2} \mathrm{H}_{5} \\
\mathrm{C}_{2} \mathrm{H}_{5} \\
\mathrm{C}_{2} \mathrm{H}_{5} \\
\mathrm{C}_{2} \mathrm{H}_{5} \\
\mathrm{C}_{2} \mathrm{H}_{5} \\
\mathrm{C}_{2} \mathrm{H}_{5} \\
\mathrm{C}_{2} \mathrm{H}_{5} \\
\mathrm{CH}\left(\mathrm{CH}_{3}\right)_{2}\end{array}$ & $\begin{array}{l}\mathbf{H} \\
\mathbf{H} \\
\mathrm{H} \\
\mathrm{OCH}_{3} \\
\mathrm{OCH}_{3} \\
\mathrm{OCH}_{3} \\
\mathrm{OCH}_{3} \\
\mathrm{OCH}_{3} \\
\mathrm{OCH}_{3}\end{array}$ & $\begin{array}{l}\left(\mathrm{CH}_{3}\right)_{2} \text { trans } \\
\left(\mathrm{CH}_{3}\right)_{2} \text { cis } \\
\left(\mathrm{C}_{2} \mathrm{H}_{5}\right)_{2} \text { cis }+ \text { trans } \\
\left(\mathrm{C}_{2} \mathrm{H}_{3}\right)_{2} \\
\left(\mathrm{C}_{2} \mathrm{H}_{5}\right)_{2} \text { cis }+ \text { trans } \\
\left(\mathrm{C}_{2} \mathrm{H}_{5}\right)_{2} \text { trans } \\
\left(\mathrm{C}_{2} \mathrm{H}_{5}\right)_{2} \text { cis (impure) } \\
\varnothing \quad \text { trans? } \\
\left(\mathrm{C}_{2} \mathrm{H}_{5}\right)_{2} \text { trans? }\end{array}$ \\
\hline
\end{tabular}

compounds by mouth. The responses to oestradiol were back to normal within 6 weeks in all groups except that receiving the highest dose of $\mathrm{H}-774$, which recovered 8 weeks after the first injection.

Results of a further test are shown in Table 3. In this test, ICI-46,474 and 47,699 were given together with $0.06 \mu \mathrm{g}$ of oestradiol, all by injection, to illustrate the lack of antagonism on first injection. Subsequently, refractoriness to oestradiol developed, as with H-774. The compounds ICI-45,692 and 45,960 had not been tested before, and so were given with and without oestradiol initially, and did not significantly affect the responses to it. Refractoriness to oestradiol developed 2 weeks after injection, and disappeared by 6 weeks. All results in Table 3 are highly significant $(P<0.01$ for inhibition by each substance during the test period).

Some of these compounds were tested by injection in spayed rats, with the results shown in Table 4. At the dose levels used, ICI-47,699 was ineffective in 
the rat but, as in mice, the other compounds failed to inhibit initial responses to oestradiol, and then caused a refractoriness to it from 2 to 4 weeks after injection, which lasted up to 8 weeks. Peak activity seemed to take rather longer to develop in the rat compared with the mouse.

Two other compounds, tested in the succeeding section because of their high antifertility activity (Collins et al., 1971) which have also caused refractoriness to $0.1 \mu \mathrm{g}$ oestradiol when tested 3 weeks after injection in mice, are H-1075

TABle 2

THE EFFECTS OF TWO CONSEGUTIVE DAILY ADMINISTRATIONS OF H-774 AND THE SEPARATED ISOMERS ON THE RESPONSE OF SPAYED MICE TO OESTRADIOL

\begin{tabular}{|c|c|c|c|c|c|c|}
\hline \multirow[t]{2}{*}{ Compound } & \multirow{2}{*}{$\begin{array}{c}\text { Total } \\
\text { dose } \\
(m g)\end{array}$} & \multirow[t]{2}{*}{ Route } & \multicolumn{4}{|c|}{$\begin{array}{c}\% \text { Positive response to } 0 \cdot 1 \mu g \text { injected } \\
\text { oestradiol after }\end{array}$} \\
\hline & & & 2 weeks & 4 weeks & 6 weeks & 8 weeks \\
\hline $\begin{array}{l}\mathrm{H}-774 \\
(\text { cis }+ \text { trans })\end{array}$ & $\begin{array}{l}0.1 \\
0.3 \\
0.9 \\
2 \cdot 7\end{array}$ & $\begin{array}{l}\text { s.c. } \\
\text { s.c. } \\
\text { s.c. } \\
\text { s.c. }\end{array}$ & $\begin{array}{r}100 \\
20 \\
0 \\
5\end{array}$ & $\begin{array}{r}100 \\
75 \\
25 \\
0\end{array}$ & $\begin{array}{l}\overline{78} \\
78 \\
11\end{array}$ & $\begin{array}{r}\overline{100} \\
78 \\
78\end{array}$ \\
\hline $\begin{array}{l}\mathrm{H}-1067 \\
(\text { trans })\end{array}$ & $\begin{array}{l}0.1 \\
0.3 \\
0.9 \\
2 \cdot 7\end{array}$ & $\begin{array}{l}\text { s.c. } \\
\text { s.c. } \\
\text { s.c. } \\
\text { s.c. }\end{array}$ & $\begin{array}{r}85 \\
5 \\
10 \\
0\end{array}$ & $\begin{array}{l}\overline{60} \\
30 \\
65\end{array}$ & $\begin{array}{l}\overline{85} \\
85 \\
89\end{array}$ & $\begin{array}{l}\overline{-} \\
\overline{-}\end{array}$ \\
\hline $\begin{array}{l}\mathrm{H}-1071 \\
(\text { cis })\end{array}$ & $\begin{array}{l}0.1 \\
0 \cdot 3 \\
0.9 \\
2 \cdot 7\end{array}$ & $\begin{array}{l}\text { s.c. } \\
\text { s.c. } \\
\text { s.c. } \\
\text { s.c. }\end{array}$ & $\begin{array}{r}100 \\
80 \\
95 \\
25\end{array}$ & $\bar{z}$ & $\frac{\overline{ }}{80}$ & $\begin{array}{l}E \\
-\end{array}$ \\
\hline H-774 & $\begin{array}{l}0.1 \\
0.3 \\
0.9 \\
2 \cdot 7\end{array}$ & $\begin{array}{l}\text { oral } \\
\text { oral } \\
\text { oral } \\
\text { oral }\end{array}$ & $\begin{array}{r}100 \\
95 \\
85 \\
75\end{array}$ & $\begin{array}{r}95 \\
100 \\
95 \\
85\end{array}$ & $\begin{array}{l}E \\
-\end{array}$ & $\begin{array}{l}- \\
-\end{array}$ \\
\hline H-1067 & $\begin{array}{l}0.1 \\
0.3 \\
0.9 \\
2.7\end{array}$ & $\begin{array}{l}\text { oral } \\
\text { oral } \\
\text { oral } \\
\text { oral }\end{array}$ & $\begin{array}{l}85 \\
95 \\
90 \\
65\end{array}$ & $\frac{-}{55}$ & $\frac{\bar{z}}{\overline{90}}$ & $\begin{array}{l}\overline{-} \\
\overline{-}\end{array}$ \\
\hline H-1071 & $\begin{array}{l}0.1 \\
0.3 \\
0.9 \\
2.7\end{array}$ & $\begin{array}{l}\text { oral } \\
\text { oral } \\
\text { oral } \\
\text { oral }\end{array}$ & $\begin{array}{l}95 \\
80 \\
95 \\
95\end{array}$ & $\bar{z}$ & $\bar{z}$ & $=$ \\
\hline Nil & vehicle & s.c. & 85 & 90 & 95 & - \\
\hline
\end{tabular}

The oestradiol was given in a vaginal smear test commencing 2, 4, 6 or 8 weeks after the first administration of the compounds. Initially, each group consisted of ten mice.

(responses to oestradiol after $0 \cdot 1,0 \cdot 3,0.9$ and $2.7 \mathrm{mg}$ were $85 \%, 85 \%, 100 \%$ and $30 \%$, respectively) and $\mathrm{H}-1076$ (responses to the same dose levels were $100 \%$, $85 \%, 65 \%$ and $15 \%$ ). Neither compound, at the same dose levels, caused refractoriness when given orally.

\section{Fertility tests}

Those compounds which have so far been tested as postcoital antifertility agents in the mouse or rat in this laboratory (all but ICI-45,692 and ICI-45,960) are very potent, causing $50 \%$ inhibition of pregnancy when given on Days 1 to 3 or 4 to 6 after mating in daily doses of from 1 to $5 \mu \mathrm{g}$. It is not surprising, 


\section{TABLE 3}

THE EFFEGTS OF TWO CONSEGUTIVE DAILY INJECTIONS OF VARIOUS IGI GOMPOUNDS ON THE RESPONSE OF SPAYED MICE TO INJEGTED OESTRADIOL

\begin{tabular}{|c|c|c|c|c|c|c|}
\hline \multirow[t]{2}{*}{ Compound } & \multirow{2}{*}{$\begin{array}{l}\text { Total } \\
\text { dose } \\
(\mathrm{mg})\end{array}$} & \multirow{2}{*}{$\begin{array}{c}\text { Dose of } \\
\text { oestradiol } \\
(\mu g)\end{array}$} & \multirow[t]{2}{*}{$\stackrel{\%}{\%}$} & \multicolumn{3}{|c|}{$\begin{array}{c}\% \text { Positive response to } 0.1 \mu g \\
\text { oestradiol after }\end{array}$} \\
\hline & & & & 2 weeks & 4 weeks & 6 weeks \\
\hline ICI-46,474 & $\begin{array}{l}0.3 \\
0.9 \\
2 \cdot 7\end{array}$ & $\begin{array}{l}0.06 \\
0.06 \\
0.06\end{array}$ & $\begin{array}{l}65 \\
75 \\
65\end{array}$ & $\begin{array}{r}75 \\
10 \\
5\end{array}$ & $\begin{array}{r}95 \\
65 \\
0\end{array}$ & $\begin{array}{l}95 \\
95 \\
40\end{array}$ \\
\hline ICI-47,699 & $\begin{array}{l}0 \cdot 3 \\
0 \cdot 9 \\
2 \cdot 7\end{array}$ & $\begin{array}{l}0.06 \\
0.06 \\
0.06\end{array}$ & $\begin{array}{r}95 \\
100 \\
100\end{array}$ & $\begin{array}{l}72 \\
30 \\
20\end{array}$ & $\begin{array}{l}69 \\
75 \\
20\end{array}$ & $\begin{array}{r}100 \\
80 \\
85\end{array}$ \\
\hline ICI-45,692 & $\begin{array}{l}0.1 \\
0.3 \\
0.9\end{array}$ & E & $\begin{array}{l}60 \\
67 \\
80\end{array}$ & $\begin{array}{l}50 \\
28 \\
15\end{array}$ & $\begin{array}{r}100 \\
67 \\
50\end{array}$ & $\begin{array}{r}95 \\
100 \\
78\end{array}$ \\
\hline ICI-45,692 & $\begin{array}{l}0 \cdot 1 \\
0 \cdot 3 \\
0 \cdot 9\end{array}$ & $\begin{array}{l}0.06 \\
0.06 \\
0.06\end{array}$ & $\begin{array}{l}78 \\
75 \\
50\end{array}$ & $\begin{array}{l}78 \\
10 \\
10\end{array}$ & $\begin{array}{r}100 \\
65 \\
25\end{array}$ & $\begin{array}{r}100 \\
85 \\
80\end{array}$ \\
\hline ICI-45,960 & $\begin{array}{l}0.1 \\
0 \cdot 3 \\
0.9\end{array}$ & E & $\begin{array}{l}30 \\
39 \\
72\end{array}$ & $\begin{array}{l}65 \\
25 \\
22\end{array}$ & $\begin{array}{r}100 \\
63 \\
31\end{array}$ & $\begin{array}{l}95 \\
81 \\
93\end{array}$ \\
\hline ICI-45,960 & $\begin{array}{l}0 \cdot 1 \\
0 \cdot 3 \\
0.9\end{array}$ & $\begin{array}{l}0.06 \\
0.06 \\
0.06\end{array}$ & $\begin{array}{l}95 \\
89 \\
65\end{array}$ & $\begin{array}{l}33 \\
39 \\
11\end{array}$ & $\begin{array}{l}61 \\
95 \\
39\end{array}$ & $\begin{array}{l}75 \\
95 \\
95\end{array}$ \\
\hline 一 & - & 0.06 & 78 & 93 & 100 & 100 \\
\hline
\end{tabular}

The oestradiol was given simultaneously with the ICI compounds or 2, 4, or 6 weeks later as in Table 2. Initially, each group consisted of ten animals.

TABLE 4

THE EFFECTS OF TWO GONSEGUTIVE DAILY INJECTIONS OF VARIOUS COMPOUNDS ON THE RESPONSE OF SPAYED RATS TO OESTRADIOL

\begin{tabular}{|c|c|c|c|c|c|c|c|}
\hline \multirow[t]{2}{*}{ Compound } & \multirow{2}{*}{$\begin{array}{l}\text { Total } \\
\text { dose } \\
(m g)\end{array}$} & \multirow{2}{*}{$\begin{array}{c}\text { Dose of } \\
\text { oestradiol } \\
(\mu \mathrm{g})\end{array}$} & \multirow[t]{2}{*}{$\begin{array}{c}\% \\
\text { Response }\end{array}$} & \multicolumn{4}{|c|}{$\begin{array}{c}\% \text { Positive response to } 0.3 \mu g \\
\text { oestradiol after }\end{array}$} \\
\hline & & & & 2 weeks & 4 weeks & 6 weeks & 8 weeks \\
\hline H-774 & $\begin{array}{l}1 \\
3 \\
9\end{array}$ & - & $\begin{array}{l}65 \\
85 \\
75\end{array}$ & $\begin{array}{l}25 \\
35 \\
50\end{array}$ & $\begin{array}{l}0 \\
0 \\
0\end{array}$ & $\begin{array}{r}15 \\
15 \\
5\end{array}$ & $\begin{array}{l}80 \\
80 \\
55\end{array}$ \\
\hline H-774 & $\begin{array}{l}1 \\
3 \\
9\end{array}$ & $\begin{array}{l}0.3 \\
0.3 \\
0.3\end{array}$ & $\begin{array}{l}70 \\
75 \\
75\end{array}$ & $\begin{array}{l}15 \\
60 \\
25\end{array}$ & $\begin{array}{r}10 \\
5 \\
0\end{array}$ & $\begin{array}{r}20 \\
50 \\
5\end{array}$ & $\begin{array}{l}75 \\
60 \\
65\end{array}$ \\
\hline IGI-46,474 & $\begin{array}{l}1 \\
3 \\
9\end{array}$ & $\begin{array}{l}0.3 \\
0.3 \\
0.3\end{array}$ & $\begin{array}{l}90 \\
85 \\
95\end{array}$ & $\begin{array}{r}100 \\
55 \\
90\end{array}$ & $\begin{array}{r}70 \\
15 \\
0\end{array}$ & $\begin{array}{l}80 \\
50 \\
20\end{array}$ & $\begin{array}{l}93 \\
83 \\
50\end{array}$ \\
\hline ICI-47,699 & $\begin{array}{l}0 \cdot 1 \\
0 \cdot 5 \\
2 \cdot 5\end{array}$ & $\begin{array}{l}0.3 \\
0.3 \\
0.3\end{array}$ & $\begin{array}{r}95 \\
100 \\
100\end{array}$ & $\begin{array}{l}50 \\
85 \\
85\end{array}$ & $\begin{array}{r}100 \\
95 \\
100\end{array}$ & $\begin{array}{r}75 \\
100 \\
90\end{array}$ & $\begin{array}{l}80 \\
90 \\
80\end{array}$ \\
\hline- & - & 0.3 & 85 & -* & 100 & 65 & 95 \\
\hline
\end{tabular}

The oestradiol was given $2,4,6$ or 8 weeks after the first administration of the compounds. Initially, each group consisted of ten animals.

* Omitted in error. 
therefore, that a dose of $1 \mathrm{mg}$ given by injection before mating should persist in effect, as shown in Table 5. It is interesting that mating was not prevented by this dose in the majority of mice, although its occurrence was delayed in some. No pregnancy followed a mating which occurred within 10 days of injection, while only $15 \%$ of matings during the next 10 days were fertile. Orally, the compounds in Table 5 were far less effective, a number of fertile matings occurring shortly after oral dosage.

TABle 5

THE EFFEGTS OF A SINGLE DOSAGE OF VARIOUS COMPOUNDS ON THE OCGURRENCE OF VAGINAL PLUGS AND RESULTING PREGNANGIES IN MICE

\begin{tabular}{|c|c|c|c|c|c|c|c|}
\hline \multirow[t]{2}{*}{ Compound } & \multicolumn{7}{|c|}{$\begin{array}{l}\text { Plugs found and litters resulting during the following } \\
\text { 10-day periods after injection: }\end{array}$} \\
\hline & & 1 to 10 & 11 to 20 & 21 to 30 & 31 to 40 & Total & $\%$ \\
\hline $\mathrm{H}-7741 \mathrm{mg}$ & $\begin{array}{l}\text { Plugs } \\
\text { Litters }\end{array}$ & $\begin{array}{r}13 \\
0\end{array}$ & $\begin{array}{l}6 \\
2\end{array}$ & $\begin{array}{l}1 \\
1\end{array}$ & $\begin{array}{l}0 \\
0\end{array}$ & $\begin{array}{r}20 \\
3\end{array}$ & $\begin{array}{r}100 \\
15\end{array}$ \\
\hline $\mathrm{H}-10751 \mathrm{mg}$ & $\begin{array}{l}\text { Plugs } \\
\text { Litters }\end{array}$ & $\begin{array}{l}7 \\
0\end{array}$ & $\begin{array}{l}6 \\
1\end{array}$ & $\begin{array}{l}4 \\
3\end{array}$ & $\begin{array}{l}1 \\
0\end{array}$ & $\begin{array}{c}18 / 19 \\
4\end{array}$ & $\begin{array}{l}95 \\
21\end{array}$ \\
\hline $\mathrm{H}-10761 \mathrm{mg}$ & $\begin{array}{l}\text { Plugs } \\
\text { Litters }\end{array}$ & $\begin{array}{r}11 \\
0\end{array}$ & $\begin{array}{l}8 \\
0\end{array}$ & $\begin{array}{l}0 \\
0\end{array}$ & $\begin{array}{l}1 \\
1\end{array}$ & $\begin{array}{r}20 \\
1\end{array}$ & $\begin{array}{r}100 \\
5\end{array}$ \\
\hline $\begin{array}{l}\text { Controls } \\
\text { vehicle only }\end{array}$ & $\begin{array}{l}\text { Plugs } \\
\text { Litters }\end{array}$ & $\begin{array}{l}19 \\
17\end{array}$ & $\begin{array}{l}1 \\
1\end{array}$ & $\begin{array}{l}0 \\
0\end{array}$ & $\begin{array}{l}\mathbf{0} \\
\mathbf{0}\end{array}$ & $\begin{array}{l}20 \\
18\end{array}$ & $\begin{array}{r}100 \\
90\end{array}$ \\
\hline \multirow[t]{2}{*}{ Compound } & \multicolumn{7}{|c|}{$\begin{array}{l}\text { Plugs found and litters resulting during the following } \\
10 \text {-day periods after oral dosage: }\end{array}$} \\
\hline & & 1 to 10 & 11 to 20 & 21 to 30 & 31 to 40 & Total & $\%$ \\
\hline $\mathrm{H}-7741 \mathrm{mg}$ & $\begin{array}{l}\text { Plugs } \\
\text { Litters }\end{array}$ & $\begin{array}{r}14 \\
5\end{array}$ & $\begin{array}{l}3 \\
3\end{array}$ & 1 & $\begin{array}{l}0 \\
0\end{array}$ & $\begin{array}{c}18 / 19 \\
9\end{array}$ & $\begin{array}{l}95 \\
47\end{array}$ \\
\hline $\mathrm{H}-10751 \mathrm{mg}$ & $\begin{array}{l}\text { Plugs } \\
\text { Litters }\end{array}$ & $\begin{array}{r}15 \\
1\end{array}$ & $\begin{array}{l}3 \\
2\end{array}$ & $\begin{array}{l}0 \\
0\end{array}$ & $\begin{array}{l}0 \\
0\end{array}$ & $\begin{array}{r}18 \\
3\end{array}$ & $\begin{array}{l}90 \\
15\end{array}$ \\
\hline $\mathrm{H}-10761 \mathrm{mg}$ & $\begin{array}{l}\text { Plugs } \\
\text { Litters }\end{array}$ & $\begin{array}{r}10 \\
2\end{array}$ & $\begin{array}{l}8 \\
5\end{array}$ & $\begin{array}{l}0 \\
0\end{array}$ & $\begin{array}{l}0 \\
0\end{array}$ & $\begin{array}{r}18 \\
7\end{array}$ & $\begin{array}{l}90 \\
35\end{array}$ \\
\hline $\begin{array}{l}\text { Controls } \\
\text { vehicle only }\end{array}$ & $\begin{array}{l}\text { Plugs } \\
\text { Litters }\end{array}$ & $\begin{array}{l}15 \\
14\end{array}$ & $\begin{array}{l}5 \\
5\end{array}$ & $\begin{array}{l}0 \\
0\end{array}$ & $\begin{array}{l}0 \\
0\end{array}$ & $\begin{array}{l}20 \\
19\end{array}$ & $\begin{array}{r}100 \\
95\end{array}$ \\
\hline
\end{tabular}

Males were introduced on Day 3 after injection. Initially, each group consisted of twenty mice.

The results of a test, in which injections of $5 \mu \mathrm{g}$ or $50 \mu \mathrm{g} /$ day were started on Day 1 and mating was allowed from the night of Day 3 were quite different (Table 6). With $5 \mu \mathrm{g}$ of H-774/day, the early matings which occurred were fertile, but sterility set in after Day 6. A dose of $50 \mu \mathrm{g} /$ day of $\mathrm{H}-774$ reduced mating frequency and all matings were sterile. With $\mathrm{H}-1075$ and $\mathrm{H}-1076$, only one litter was produced in any group, although the mating frequency with the latter compound at $5 \mu \mathrm{g} /$ day was not significantly less than in controls.

An attempt to repeat the test in Table 6 by oral administration was unsuccessful, since a daily dose of $0.1 \mathrm{ml}$ of $50 \%$ aqueous propylene glycol reduced mating in controls to $30 \%$ over the test period, and litters to $15 \%$. The mice swelled 
visibly with intestinal gases and then recovered, even with continued administration, but the results of the test were clearly valueless. Further oral testing will be attempted at a later date using a different medium.

\section{TABLE 6}

THE EFFECTS OF THE INJECTION OF VARIOUS COMPOUNDS ON THE OCGURRENCE OF VAGINAL PLUGS AND RESULTING PREGNANGIES IN MICE

\begin{tabular}{|c|c|c|c|c|c|c|c|c|c|c|c|}
\hline \multirow[t]{2}{*}{ Compound } & \multirow{2}{*}{$\begin{array}{c}\text { Dose } \\
(\mu g / \text { day })\end{array}$} & \multicolumn{10}{|c|}{$\begin{array}{l}\text { Plugs found and litters resulting during the } \\
\text { following single days after the start of injections: }\end{array}$} \\
\hline & & & 4 & 5 & 6 & 7 & 8 & 9 & 10 & Total & $\%$ \\
\hline \multirow[t]{2}{*}{$\mathrm{H}-774$} & 5 & $\begin{array}{l}\text { Plugs } \\
\text { Litters }\end{array}$ & $\begin{array}{l}4 \\
4\end{array}$ & $\frac{1}{1}$ & 1 & $\begin{array}{l}9 \\
1\end{array}$ & $\begin{array}{l}1 \\
0\end{array}$ & $\begin{array}{l}2 \\
0\end{array}$ & $\begin{array}{l}0 \\
0\end{array}$ & $\begin{array}{r}18 \\
7\end{array}$ & $\begin{array}{l}90 \\
35\end{array}$ \\
\hline & 50 & $\begin{array}{l}\text { Plugs } \\
\text { Litters }\end{array}$ & $\begin{array}{l}4 \\
0\end{array}$ & $\begin{array}{l}0 \\
0\end{array}$ & $\begin{array}{l}0 \\
0\end{array}$ & $\begin{array}{l}0 \\
0\end{array}$ & $\begin{array}{l}3 \\
0\end{array}$ & $\begin{array}{l}5 \\
0\end{array}$ & $\begin{array}{l}0 \\
0\end{array}$ & $\begin{array}{r}12 \\
0\end{array}$ & $\begin{array}{r}60 \\
0\end{array}$ \\
\hline \multirow[t]{2}{*}{ H-1075 } & 5 & $\begin{array}{l}\text { Plugs } \\
\text { Litters }\end{array}$ & $\begin{array}{l}4 \\
0\end{array}$ & $\begin{array}{l}3 \\
0\end{array}$ & $\begin{array}{l}1 \\
0\end{array}$ & $\begin{array}{l}2 \\
0\end{array}$ & $\begin{array}{l}1 \\
0\end{array}$ & $\begin{array}{l}2 \\
0\end{array}$ & $\begin{array}{l}0 \\
0\end{array}$ & $\begin{array}{r}13 \\
0\end{array}$ & $\begin{array}{r}65 \\
0\end{array}$ \\
\hline & 50 & $\begin{array}{l}\text { Plugs } \\
\text { Litters }\end{array}$ & $\begin{array}{l}2 \\
0\end{array}$ & $\begin{array}{l}3 \\
0\end{array}$ & $\begin{array}{l}1 \\
0\end{array}$ & $\begin{array}{l}2 \\
0\end{array}$ & $\begin{array}{l}1 \\
0\end{array}$ & $\begin{array}{l}1 \\
0\end{array}$ & $\begin{array}{l}0 \\
0\end{array}$ & $\begin{array}{r}10 \\
0\end{array}$ & $\begin{array}{r}50 \\
0\end{array}$ \\
\hline \multirow[t]{2}{*}{$\mathrm{H}-1076$} & 5 & $\begin{array}{l}\text { Plugs } \\
\text { Litters }\end{array}$ & $\begin{array}{r}10 \\
1\end{array}$ & $\begin{array}{l}1 \\
0\end{array}$ & $\begin{array}{l}0 \\
0\end{array}$ & $\begin{array}{l}3 \\
0\end{array}$ & $\begin{array}{l}2 \\
0\end{array}$ & $\begin{array}{l}0 \\
0\end{array}$ & $\begin{array}{l}0 \\
0\end{array}$ & $\underset{1}{16 / 19}$ & $\begin{array}{r}84 \\
5\end{array}$ \\
\hline & 50 & $\begin{array}{l}\text { Plugs } \\
\text { Litters }\end{array}$ & $\begin{array}{l}2 \\
0\end{array}$ & $\begin{array}{l}1 \\
0\end{array}$ & $\begin{array}{l}1 \\
0\end{array}$ & $\begin{array}{l}2 \\
0\end{array}$ & $\begin{array}{l}3 \\
0\end{array}$ & $\begin{array}{l}1 \\
0\end{array}$ & $\begin{array}{l}1 \\
0\end{array}$ & $\begin{array}{r}11 \\
0\end{array}$ & $\begin{array}{r}55 \\
0\end{array}$ \\
\hline Controls & 0 & $\begin{array}{l}\text { Plugs } \\
\text { Litters }\end{array}$ & $\begin{array}{l}5 \\
5\end{array}$ & $\begin{array}{l}2 \\
2\end{array}$ & $\begin{array}{l}4 \\
4\end{array}$ & $\begin{array}{l}7 \\
6\end{array}$ & $\begin{array}{l}1 \\
1\end{array}$ & $\begin{array}{l}0 \\
0\end{array}$ & $\begin{array}{l}0 \\
0\end{array}$ & $\begin{array}{l}19 \\
18\end{array}$ & $\begin{array}{l}95 \\
90\end{array}$ \\
\hline
\end{tabular}

Injections were begun on Day 1 and males were introduced on Day 3. Initially, each group consisted of twenty mice.

\section{DISCUSSION}

Harper \& Walpole (1966) found that ICI-46,474 and ICI-47,699 have antifertility activities which, in the mouse, can be explained by their oestrogenic potencies. Collins et al. (1971) feel that neither with these compounds, nor with others of the series, can frank oestrogenicity be adequate to explain the high antifertility potencies observed, and that dissociation of these properties is seen in the series. The absence of antioestrogenic properties, as tested both by Harper \& Walpole (1966) and by Collins et al. (1971), naturally led these authors to neglect such properties as contributing to antifertility effects in the mouse, although ICI 46,474 is strongly antioestrogenic in the rat. The recent suggestion of Emmens \& Miller (1969) that at least some types of antioestrogen owe this property to their being short-acting oestrogens which displace others from the site of action and then do not continue to act themselves, would make it feasible that an antifertility compound could well show oestrogenic properties in one species and antioestrogenic properties in another, and yet act as an antifertility agent by the same mechanism in both. Terenius (1970) has now demonstrated that meso-butoestrol is a short-acting compound, behaving as an antioestrogen only in local tests or in tests in vitro, whereas $\mathrm{U}-11,100 \mathrm{~A}$ remains at receptor sites and acts as a systemic antioestrogen. 
The effects of triarylalkenes as antifertility agents when administered either immediately or shortly after coitus, and their longer-term effects when given before coitus might well be due to the same mechanism, particularly since high doses were given in the tests showing infertility for 10 or more days. Such doses might well have stayed in the body or at the site of injection for this period. However, if either oestrogenic or anti-oestrogenic activity is, in fact, directly involved, antifertility effects must be supposed to depend on first one and then the other as time goes on. The period of infertility after injection of H-774 lasted for 20 or more days (Table 5), one third or less matings resulting in litters. The period of infertility after oral dosage was quite brief. The periods of antioestrogenic activity after injection and oral dosage (Table 2) were similar to those of infertility. Since the route of administration affects both types of result, it seems likely that the compound stays around for longer when injected and gives rise to both effects, whether or not these are due to the same mechanism.

The first problem suggested by these observations is an investigation of the long-term effects of the triarylalkenes on the physiology and morphology of the female reproductive tract. The second is a fuller investigation of the infertility resulting from dosage before mating, with better estimates of its duration and nature. These are both under investigation.

\section{ACKNOWLEDGMENTS}

I wish to thank Dr Walpole for the provision of the ICI compounds used, and Dr Collins and Dr Hobbs of this department, who made the $\mathrm{H}$ compounds. This work was assisted by a grant from the Ford Foundation.

\section{REFERENCES}

Collins, D. J., Hobrs, J. J. \& Emmens, C. W. (1971) Antiestrogenic and antifertility compounds. IV. 1,1,2-triarylalkan-1-ols and 1,1,2-triarylalk-1-enes containing basic ether groups. 7 . medicinal Chem. (in press).

Emmens, C. W. (1969) Estrogens. In: Methods in Hormone Research, Vol. 2A, p. 61. Ed. R. I. Dorfman. Academic Press, New York.

Emmens, C. W. (1970) Antifertility agents. A. Rev. Pharmac. 10, 237.

Emmens, C. W., Humphrey, K., Martin, L. \& Owen, W. H. (1967) Antifertility properties of two non-oestrogenic steroids and MRL-37. Steroids, 9, 235.

Emmens, G. W. \& Miller, B. G. (1969) Estrogens, proestrogens and antiestrogens. Steroids, 13, 725.

HARPER, M. J. K. \& WALPOLE, A. L. (1966) Contrasting endocrine activities of cis and trans isomers in a series of substituted triphenylethylenes. Nature, Lond. 212, 87.

Lerner, L. J., Holthaus, F. J. \& Thompson, C. R. (1958) A non-steroidal oestrogen antagonist 1-(p-2-diethylaminoethoxyphenyl)-1-phenyl-2-p-methoxyphenyl ethanol. Endocrinology, 63, 295.

Terentus, L. (1970) Two modes of interaction between oestrogen and anti-oestrogen. Acta endocr., Copenh. 64, 47. 